\title{
Deriving the Metallicity Distribution Function of Galactic Systems
}

\author{
Yeshe Fenner and Brad K. Gibson \\ Centre for Astrophysics \& Supercomputing, Swinburne University, Mail \#31, Victoria 3122, Australia \\ yfenner@astro.swin.edu.au \\ bgibson@astro.swin.edu.au \\ Received 2002 November 19, accepted 2003 April 4
}

\begin{abstract}
The chemical evolution of the Milky Way is investigated using a dual-phase metal-enriched infall model in which primordial gas fuels the earliest epoch of star formation, followed by the ongoing formation of stars from newly accreted gas. The latest metallicity distribution of local K-dwarfs is reproduced by this model, which allows the Galactic thin disk to form from slightly metal-enriched gas with $\alpha$-element enhancement. Our model predicts ages for the stellar halo and thin disk of 12.5 and $7.4 \mathrm{Gyr}$ respectively, in agreement with empirically determined values. The model presented in this paper is compared with a similar dual-phase infall model from Chiappini et al. (2001). We discuss a degeneracy that enables both models to recover the K-dwarf metallicity distribution while yielding different star formation histories.

The metallicity distribution function (MDF) of K-dwarfs is proposed to be more directly comparable to chemical evolution model results than the G-dwarf distribution because lower mass K-dwarfs are less susceptible to stellar evolutionary effects. The K-dwarf MDF should consequently be a better probe of star formation history and provide a stronger constraint to chemical evolution models than the widely used G-dwarf MDF. The corrections that should be applied to a G-dwarf MDF are quantified for the case of the outer halo of NGC 5128.
\end{abstract}

Keywords: galaxy: solar neighbourhood, evolution — stars: abundances

\section{Introduction}

The metallicity distributions of stars in different environments yield important information about the age and formation history of stellar systems. Particular attention has been paid to the metallicity distribution function (MDF) of G-dwarfs in the solar neighbourhood, as this provides one of the strongest constraints on Galactic chemical evolution models. These models solve the sets of differential equations governing the formation and destruction of the elements through stellar processes (Tinsley 1980). The over-prediction of low-metallicity stars, the so-called 'G-dwarf problem', is typically resolved by allowing the disk of the Milky Way to form gradually from accreting gas on a timescale of about 7 Gyr. Recent models (e.g. Chiappini et al. 1997; Goswami \& Prantzos 2000) have successfully reproduced the observed G-dwarf MDF using dual-phase accretion models in which the halo/thick disk component evolves independently of the thin disk and on a rapid timescale.

An implicit assumption of most theoretically predicted G-dwarf metallicity distributions is that all G-dwarfs have lifetimes older than the age of the Milky Way. In fact, the stars in the Rocha-Pinto \& Maciel (1996) sample have masses ranging from $0.7 \mathrm{M}_{\odot}$ to $1.1 \mathrm{M}_{\odot}$, while Wyse \& Gilmore's (1995) dataset spans $0.8 \mathrm{M}_{\odot}$ to $1.2 \mathrm{M}_{\odot}$. Some of these stars have lifetimes shorter than the age of the disk. Furthermore, the earliest formed G-dwarfs will have even shorter lifetimes owing to their low metallicity, which reduces their opacity and raises their luminosity. For instance, a $1.3 \mathrm{M}_{\odot}$ star leaves the main sequence after about $4 \mathrm{Gyr}$ at solar metallicity, or after less than $3 \mathrm{Gyr}$ for $\mathrm{Z}=0.05 \mathrm{Z}_{\odot}$ (Schaller et al. 1992). In the low-metallicity environment of the early Milky Way, a $1 \mathrm{M}_{\odot}$ star is expected to leave the main sequence after about $6 \mathrm{Gyr}$. The preferential loss of the oldest G-dwarfs from empirical datasets tends to bias the MDF towards higher metallicity and suppresses the low-metallicity tail. However, the protracted formation history of the Galactic disk means that stellar lifetime effects alone cannot account for the paucity of metal-poor G-dwarfs with respect to 'closed box' models of chemical evolution. We have investigated the impact of these effects on the shape and peak of measured MDFs in different stellar environments, the results of which are presented in Section 4.2. Rocha-Pinto \& Maciel (1997) and Bazan \& Mathews (1990) should be referred to for additional commentary in this regard.

Since K-dwarfs are less massive than G-dwarfs, their lifetimes are longer than the age of the Galaxy. The Kdwarf metallicity distribution should therefore trace the 'true' MDF because surveys of this stellar population should not suffer from incompleteness due to stars evolving off the main sequence. Because of their faintness, however, accurate metallicities of large numbers of Kdwarfs have only recently been obtained. Kotoneva et al. (2002) constructed a K-dwarf distribution using a sample of 220 nearby K-dwarfs drawn from the Hipparcos catalogue (ESA 1997). They were able to select K-dwarfs by absolute magnitude and restrict the mass range to $0.7-0.9$ $\mathrm{M}_{\odot}$, for which evolutionary effects are unimportant.

The 'Galaxy Evolution tool' (Fenner \& Gibson in preparation) - GEtool $^{1}$ — was used to numerically solve

\footnotetext{
${ }^{1}$ http://astronomy.swin.edu.au/GEtool/
} 
the equations governing the rate of change of gas mass and elemental abundances due to star formation, supernovae feedback, and the continual accretion of gas onto the disk. The model presented here is capable of reproducing the main observed properties of the Milky Way, including the present-day radial gas distribution, radial abundance gradients, age-metallicity relation, stellar metallicity distribution and elemental abundance ratios. Section 2 outlines the features of the model, while Section 3 describes the adopted stellar nucleosynthetic prescriptions. The predicted K-dwarf metallicity distribution is presented in Section 4.1, where it is compared with the measured K-dwarf MDF in order to infer the possible formation history of the Galaxy. Section 4.2 shows results from a study into the effect of finite stellar lifetimes on the measured MDFs of populations in different star forming environments.

\section{The Model}

\subsection{Infall Scheme}

Our Galactic evolution model assumes two main formation phases. The formation of halo stars is associated with the first episode of infalling material, while the second phase leads to disk formation. To aid the comparison with other dual-phase Galactic chemical evolution studies the model is restricted to simulate two rather than three infall phases. The thick disk is assumed to correspond to a merger-heated thin disk and is therefore chiefly associated with the early stages of the second formation epoch. The difficulty inherent in using this kind of dual-phase model to describe a three component system is further discussed in Section 4.1. Simulating the evolution of a separate thick disk component will be explored in forthcoming work.

The initial phase of star formation occurs on a timescale of $\sim 0.5 \mathrm{Gyr}$ and enriches the initially primordial gas to a metallicity of $[\mathrm{Fe} / \mathrm{H}] \approx-1.2$. The second formation phase is delayed by $1 \mathrm{Gyr}$ with respect to the first phase and has a more protracted star formation history; high velocity clouds (HVCs) may represent the present-day source of this Galactic star formation fuel. Drawing on observations of the chemical composition of these clouds (Wakker et al. 1999; Gibson et al. 2001; Sembach et al. 2002), the second accretion phase assumes the gas is slightly metalenriched $\left(Z=0.1 Z_{\odot}\right)$ with an enhancement of $\alpha$-elements relative to iron. The adopted value of $[\alpha / \mathrm{Fe}]=+0.4$ is consistent with $\alpha / \mathrm{Fe}$ ratios found in metal-poor stars (e.g. Ryan, Norris \& Beers, 1996). The precise dependence of key observational constraints on the assumed chemical composition of the infalling gas will be presented in a forthcoming paper (Fenner \& Gibson in preparation).

After Chiappini et al. (1997), we assume that the rate at which material is accreted during these phases declines exponentially. The evolution of total surface mass density $\sigma_{\text {tot }}(r, t)$ is given by

$$
\frac{d \sigma_{\mathrm{tot}}(r, t)}{d t}=A(r) e^{-t / \tau_{\mathrm{H}}(r)}+B(r) e^{-\left(t-t_{\text {delay }}\right) / \tau_{\mathrm{D}}(r)}
$$

where the infall rate coefficients $A(\mathrm{r})$ and $B(\mathrm{r})$ are chosen in order to reproduce the present-day surface mass density of the halo and disk components, which we take to be 10 and $45 \mathrm{M}_{\odot} \mathrm{pc}^{-2}$ respectively. These values are comparable with 17 and $54 \mathrm{M}_{\odot} \mathrm{pc}^{-2}$ used by Chiappini et al. (2001). The adopted timescales for the infall phases are $\tau_{\mathrm{H}}=0.5 \mathrm{Gyr}$ and $\tau_{\mathrm{D}}=7.0 \mathrm{Gyr}$ at the solar radius $r_{\odot}=8 \mathrm{kpc}$. The 'inside-out' functional form for $\tau_{\mathrm{D}}(r)$ (Romano et al. 2000) is adopted, with the Milky Way age taken to be $13 \mathrm{Gyr}$.

\subsection{Star Formation Formalism}

We adopt a star formation prescription which is based upon the hypothesis that star formation is triggered by the compression of interstellar material from spiral arm motion (e.g. Prantzos \& Silk 1998). This implies that the star formation rate (SFR) is proportional to the angular frequency of the spiral pattern and therefore inversely proportional to radius. The star formation law incorporated in this model is given by

$$
\psi(r, t)=v \sigma_{\mathrm{gas}}^{2}(r, t)\left(\frac{r_{\odot}}{r}\right)
$$

where the value of the efficiency factor $v$ is constrained by the present-day gas fraction.

\subsection{Initial Mass Function}

The shape of the stellar initial mass function (IMF) influences the quantity of Galactic material locked up in stars of different masses, which in turn determines the rate at which different elements are released into the interstellar medium. The models presented in Section 4.1 use the Kroupa et al. (1993) three-component IMF. With respect to the Salpeter (1955) and Scalo (1986) IMFs, this function has a steeper slope for high masses, resulting in less material being processed by massive stars. We impose an upper mass limit of $60 \mathrm{M}_{\odot}$ on stellar formation in order to recover the observed trend of $[\mathrm{O} / \mathrm{Fe}]$ at low metallicity (e.g. Carretta et al. 2000, Melendez et al. 2001), while avoiding the overproduction of oxygen.

\subsection{Properties of the Halo, Thick Disk and Thin Disk Components}

The ratio of the local number density of thick disk to thin disk stars lies between $1 \%$ and $6 \%$ (Gilmore et al. 1995). Both disk components are well fitted in vertical height, $z$, with exponential functions. Taking the thick and thin disk scale heights to be $1 \mathrm{kpc}$ and $330 \mathrm{pc}$ respectively (Gilmore et al. 1995), the ratio of the thick to thin disk surface densities lies between $2 \%$ and $20 \%$. The local stellar density of halo stars relative to thin disk stars is only $\sim 0.1 \%$ with a mean metallicity of $[\mathrm{Fe} / \mathrm{H}]=-1.7$ (Norris \& Ryan 1991), while thick disk stars have typical metallicities in the interval $-0.4<[\mathrm{Fe} / \mathrm{H}]<-1.2$ with a mean of -0.7 (Gilmore et al. 1995). In comparison, the MDF of thin disk stars peaks near $[\mathrm{Fe} / \mathrm{H}]=-0.2$ (McWilliam 1997). In addition to metallicity, kinematic behaviour is used to 
distinguish between these three components. Thick disk kinematics lie somewhere between those of the halo and the thin disk, and describe a distinct population.

\section{Yields}

The observed behaviour of elemental ratios such as $[\mathrm{O} / \mathrm{Fe}]$ with $[\mathrm{Fe} / \mathrm{H}]$ are important observational constraints on chemical evolution models. Oxygen is chiefly produced in massive stars, whereas a significant fraction of iron is supplied by Type Ia supernovae involving binary systems of lower mass stars. The characteristic timescale on which oxygen is ejected into the ISM is therefore much shorter than that of iron, owing to the mass dependence of main sequence lifetimes.

In order to recover the observed behaviour of elemental abundance ratios, the instantaneous recycling approximation has been relaxed. Mass and metallicity-dependent lifetimes were taken from Schaller et al. (1992).

\subsection{Massive Stars}

The present work incorporates an updated set of stellar yields from Limongi et al. (2000; 2002), supplemented with a finer mass coverage grid (kindly provided by Marco Limongi), covering a range of stellar initial masses and metallicities $\left(13<m / \mathrm{M}_{\odot}<80\right.$ and $\left.\mathrm{Z} / \mathrm{Z}_{\odot}=0,10^{-3}, 1\right)$. Stellar yields are one of the most important ingredients in Galactic chemical evolution models, yet questions remain concerning the composition of ejected material, owing to the uncertain role played by processes such as mass loss, rotation, fall-back, and the location of the mass cut in supernovae, which separates the remnant from the ejected material.

The amount of iron released in supernova explosions of massive stars is particularly sensitive to fall-back and the location of the mass cut. There are few supernova observations with which to directly infer the amount of iron ejected. We have therefore taken the opposite approach of using the observed trend of abundance ratios to indirectly constrain iron yields. The Limongi et al. (2000; 2002) yields are presented for a range of mass cuts, allowing a self-consistent treatment of all yields (c.f. Goswami \& Prantzos 2000; Argast et al. 2002). We have found that the trend of $[\mathrm{O} / \mathrm{Fe}]$ with $[\mathrm{Fe} / \mathrm{H}]$ is best recovered by suppressing the ejection of iron in stars more massive than about $30 \mathrm{M}_{\odot}$. This leads to a smaller contribution to the interstellar iron abundance from Type II supernovae than is obtained from the canonical Woosley \& Weaver (1995) model. A preliminary relationship between the mass cut and iron yield was presented in Fenner, Gibson \& Limongi (2002); a full accounting will be provided in Fenner \& Gibson (in preparation).

\subsection{Low- and Intermediate-Mass Stars}

The metallicity-dependent yields of Renzini \& Voli (1981) were used for stars in the $1 \leq m / \mathrm{M}_{\odot} \leq 8$ range. Yields for stars with masses between $8 \mathrm{M}_{\odot}$ and $13 \mathrm{M}_{\odot}$ were estimated by interpolating between the highest mass in
Renzini \& Voli and the lowest mass in Limongi et al (2000; 2002). It should be noted, however, that it is not yet clear whether stars in the range $8<m / \mathrm{M}_{\odot}<13$ contribute to interstellar enrichment, or whether the processed material becomes trapped in the remnant following core collapse.

\subsection{Type Ia Supernovae}

The yields for Type Ia SNe were taken from the W7 model of Thielemann et al. (1993). The fraction of binaries resulting in Type Ia supernovae was fixed by requiring that the model reproduce the local age-metallicity relation; a fraction of 3\% was adopted, leading to model predictions consistent with observation. Type Ia supernovae supply about one half of the interstellar iron abundance in this model.

\section{Results}

\subsection{The Solar Neighbourhood}

The predicted star formation rate in the solar vicinity (solid line) is shown as a function of time in the upper panel of Figure 1 along with the range of measured values (vertical bar). The star formation rate in the halo peaks at almost $3 \mathrm{M}_{\odot} \mathrm{pc}^{-2} \mathrm{Gyr}^{-1}$ after $500 \mathrm{Myr}$. The predicted present-day rate of star formation in the thin disk of $2.7 \mathrm{M}_{\odot} \mathrm{pc}^{-2} \mathrm{Gyr}^{-1}$ agrees with observations (Chiappini et al. 2001) and is about 2.5 times lower than the maximum rate of star formation which occurred $\sim 10$ Gyr ago, 2-3 Gyr after the onset of the second infall phase.

The dotted line in the upper panel of Figure 1 depicts the star formation rate given by the Chiappini et al. (2001) dual-phase Galactic chemical evolution model. Like the Milky Way model presented in this paper, the Chiappini et al. model is consistent with many observational constraints, including the Kotoneva et al. (2002) empirical MDF. Despite both models satisfying key empirical constraints such as the K-dwarf metallicity distribution, they do predict very different properties for the halo, thick disk, and thin disk components of the Milky Way. This is largely a consequence of different star formation rates in the earliest epoch of Galactic evolution. The predicted number of stars born during the first billion years differs by a factor of 2.5 between the two models, whereas the total number of stars formed differs by less than $20 \%$. The higher initial rate of star formation in Chiappini et al. leads to a more rapid enrichment of the interstellar medium, when compared with the model presented here. The implications of these differences in terms of the Milky Way's stellar components are quantified further below.

First, the model we have employed here is successful in reproducing the new Kotoneva et al. (2002) K-dwarf MDF, as shown in the lower panel of Figure 1. The predicted MDF has been convolved with a $\sigma=0.1$ dex Gaussian in $[\mathrm{Fe} / \mathrm{H}]$, consistent with the observational uncertainties in the data. Plotted against the model results (solid curve) is the K-dwarf MDF from Kotoneva et al. (squares with error bars) and the G-dwarf MDF from Hou et al. (1998) (dotted line histogram). The model MDF provides 

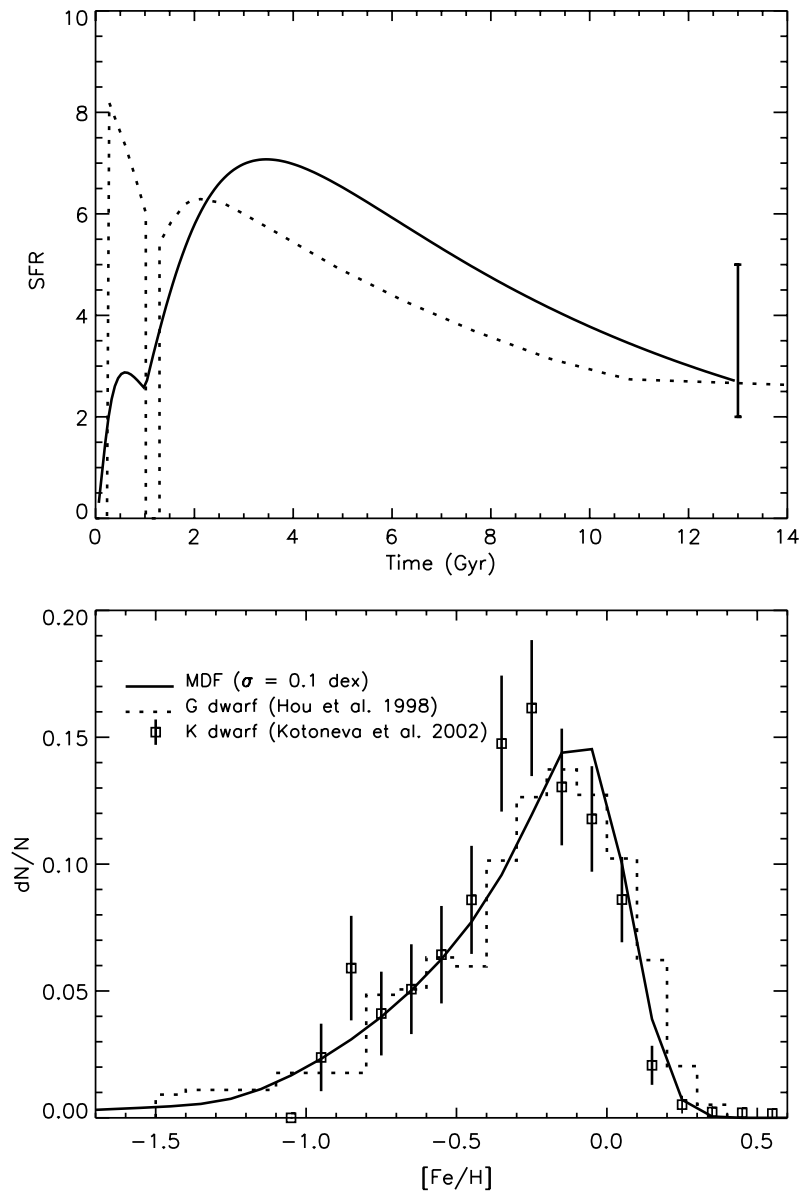

Figure 1 Upper panel: Evolution of the star formation rate (SFR) predicted by the dual-phase infall model described here (solid line). The dotted line illustrates the stronger initial burst of star formation in the Chiappini et al. (2001) dual-infall model. While the total integrated star formation differs between the two models by less than $20 \%$, during the first $1 \mathrm{Gyr}$ the Chiappini et al. model supplies $\sim 2.5$ times more stars than in model. The SFR in the Chiappini et al. disk phase also peaks $1.5 \mathrm{Gyr}$ earlier than in our model. The vertical bar denotes the observed limit on the present-day value. Lower panel: The calculated K-dwarf distribution (solid curve) is plotted against the datasets of Hou et al. (1998 - dotted histogram), and Kotoneva et al. (2002 - open squares). The theoretical MDF has been convolved with a Gaussian of dispersion $\sigma=0.1 \mathrm{dex}$ in $[\mathrm{Fe} / \mathrm{H}]$, consistent with the known empirical uncertainties.

a satisfactory agreement with the Kotoneva et al. dataset, although the model peaks $\sim 0.2 \mathrm{dex}$ higher in $[\mathrm{Fe} / \mathrm{H}]$. The model also follows closely the Hou et al. G-dwarf MDF, which exhibits a longer metal-poor tail than the $\mathrm{K}$-dwarf distribution. While most authors measure the peak of the local G-dwarf metallicity distribution to lie between -0.2 and -0.3 dex (e.g. Wyse \& Gilmore 1995; Rocha-Pinto \& Maciel 1996), there is still not complete agreement. Haywood (2001) found that the MDF of a colour-selected sample was centered on solar metallicity, however Kotoneva et al. showed that a bias toward metalrich stars might arise due to a systematic trend between metallicity distribution and stellar colour.

The complete absence of stars with $[\mathrm{Fe} / \mathrm{H}]<-1$ in the Kotoneva et al. (2002) sample is not reproduced by our model (solid curve). In Chiappini et al.

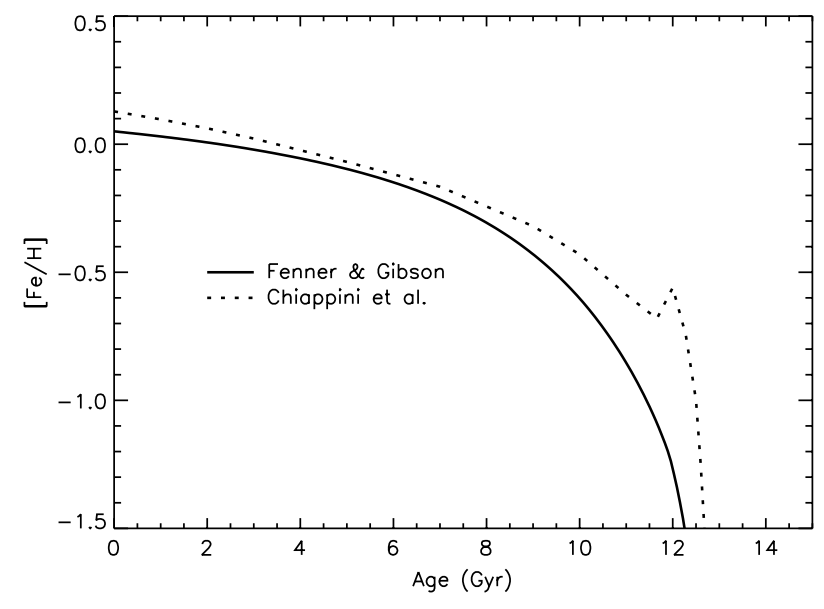

Figure 2 The age-metallicity relation (AMR) in the solar neighbourhood predicted by our model (solid curve) and by Chiappini et al. (2001 - dotted line). While similar present-day iron abundances are predicted by both models, in Chiappini et al. the ISM is polluted on a much shorter timescale. Our model assumes a Galactic age of $13 \mathrm{Gyr}$, whereas Chiappini et al. (2001) take a value of $14 \mathrm{Gyr}$. In comparing the two AMRs, we have shifted the Chiappini et al. curve by $1 \mathrm{Gyr}$ such that both models span $13 \mathrm{Gyr}$.

(1997; 2001) and Kotoneva et al. (2002), any lowmetallicity tail in the theoretical MDF is suppressed by neglecting either: (i) stars with $[\mathrm{Fe} / \mathrm{H}]<-1.2$ (e.g. Chiappini et al. 1997; Kotoneva et al. 2002); or (ii) stars born before $\mathrm{t}=1 \mathrm{Gyr}$ (which effectively ignores stars with $[\mathrm{Fe} / \mathrm{H}]<-0.6)$ (e.g. Chiappini et al. 2001). In comparison, we have included all long-lived stars in the metallicity distribution function. Stars with $[\mathrm{Fe} / \mathrm{H}]<-1.2$ represent a very small fraction of present-day stars in the Chiappini et al. model due to their strong initial burst of star formation. Efficient, early star formation leads to a metallicity $[\mathrm{Fe} / \mathrm{H}]=-1.2$ being reached on a very short timescale $(<0.1 \mathrm{Gyr})$. Such rapid initial enrichment is one of the main differences between the present model and that of Chiappini et al. We assume a more moderate initial phase of star formation, leading to a metal-poor tail (i.e. $[\mathrm{Fe} / \mathrm{H}]<-1.2$ ) in the MDF which contains $\sim 4 \%$ of the stars. The age associated with this metal-poor tail is $12.5 \mathrm{Gyr}$, while the median age of our predicted thin disk dwarfs is 7.5 Gyr. This is in excellent agreement with Hansen et al. (2002) who derive ages of $7.3 \pm 1.5 \mathrm{Gyr}$ and $12.7 \pm 0.7$ Gyr for Galactic thin disk white dwarfs and for the halo globular cluster M4 respectively.

The age-metallicity relation (AMR) for our dual-infall model is shown in Figure 2 (solid curve). It takes $\sim 1 \mathrm{Gyr}$ to enrich the interstellar medium in the solar neighbourhood to a metallicity of $[\mathrm{Fe} / \mathrm{H}]=-1.25$. In contrast, the Chiappini et al. (2001) model (dotted curve) reaches the much greater metallicity of $[\mathrm{Fe} / \mathrm{H}] \approx-0.5$ over the same time interval. The AMR from Chiappini et al. (2001) differs from that in the 1997 version of their two-infall model, owing to different timescales for the first episode of infall. Their halo/thick disk accumulates on a timescale of $2 \mathrm{Gyr}$ in the 1997 model, with a $2 \mathrm{Gyr}$ delay before the thin disk starts to form. In the later model, these 
timescales are shortened to $\sim 1 \mathrm{Gyr}$, giving rise to an even steeper AMR than in the 1997 paper. The kink seen in the Chiappini et al. AMR is due to the metallicity of the ISM becoming diluted when the second phase of infall commences at $1 \mathrm{Gyr}$. It is not until the thin disk gas surface density reaches a threshold of $7 \mathrm{M}_{\odot} \mathrm{pc}^{-2}$ that Chiappini et al. allow thin disk stars to form. No such star formation threshold is incorporated in our model.

We wish to stress that caution must be employed when drawing conclusions regarding the nature of three stellar population components of the Milky Way (thin disk, thick disk, halo), when the models are inherently limited by their dual-infall nature. That said, if one adopts a chemical criterion of $-1.0<[\mathrm{Fe} / \mathrm{H}]<-0.5$ for the definition of 'thick disk', then the age of the thick disk predicted under the Chiappini et al. formalism would be centered upon $12.5 \mathrm{Gyr}$, with a vanishingly small age-spread $(<0.2 \mathrm{Gyr})$, and possess kinematics closely aligned with the first of the infall phases (i.e. the halo). Conversely, the thick disk predicted under our formalism would be 2-3 Gyr younger, with an age-spread an order of magnitude larger ( $\sim 2 \mathrm{Gyr})$, and possess kinematics more closely resembling those of the second infall phase (i.e. the disk). The Kotoneva et al. (2002) sample of solar neighbourhood K-dwarfs identified only two of 431 stars as having halo kinematics. Thus halo stars are rare, whereas the Chiappini model would associate a large fraction of stellar mass with the halo component.

In the Chiappini et al. model of Galaxy formation, the first infall phase is associated with the formation of the halo and thick disk. Thus these components are coeval and form prior to, and independently of, the thin disk. In such a scenario, the thick disk would have little dispersion in age, and the stars would exhibit primarily halo kinematics. The model presented here associates the first infall phase with the build-up of the halo. In contrast with Chiappini et al., the formation of the thick disk overlaps with the early evolution of the thin disk (during the second infall phase). Our thick disk, as defined by metallicity, would have predominantly disk-like kinematics and a broader range of ages.

While both formalisms clearly have problems in describing a three-component system, we favour the scenario presented in this paper, in which the intermediate metallicity population (thick disk) has a typical age several gigayears younger than the halo (as inferred by observation - e.g. Chayboyer et al. 2000; Ibukiyama \& Arimoto 2002) and kinematics akin to that of a disk-like population, and not a rapid-collapse halo population. In that sense, our model is more consistent with the favoured model of Galactic thick disk formation whereby an existing stellar thin disk 'puffs up' during the process of a merger (e.g. Wyse 2001).

\subsection{Lifetime Effects and MDFs in External Galaxies: A Note of Caution}

Recent progress has been made in measuring the metallicity distribution in the stellar halos of external galaxies

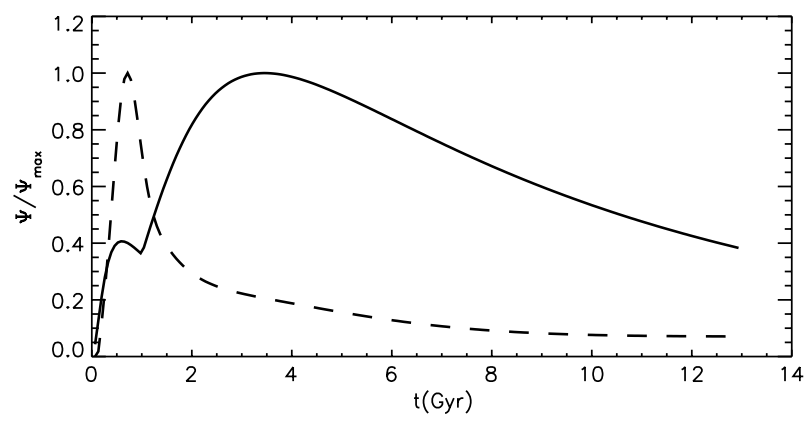

Figure 3 Evolution of the star formation rate in a typical halo chemical evolution model (dashed curve) and in the Milky Way model (solid curve).

(e.g. Durrell et al. 2001; Harris \& Harris 2000, 2002). The observed metallicity distribution of these systems, which presumably formed early and on a short timescale, is likely to be biased by the effect of finite stellar lifetimes. In an attempt to quantify the discrepancy between the observed and 'true' MDF due to lifetime effects, we constructed a 'toy' model of the NGC 5128 halo using the chemical evolution code described in this paper. This NGC 5128 halo model has some key differences with respect to the Milky Way model described in this paper. The main differences are: a single phase of star formation; a shorter formation timescale ( $\sim 1 \mathrm{Gyr})$; and initial composition of gas is primordial.

In stellar systems such as halos, a significant fraction of stars classified as G-dwarfs may have evolved off the main sequence. This has the effect of preferentially removing older and therefore more metal-poor stars from the observed present-day G-dwarf population. In a similar way, the metal-poor tail in the measured G-dwarf MDF is further suppressed by the shorter lifetimes of low mass, metal-poor stars (e.g. Schaller et al. 1992). On the other hand, the disk of our own Galaxy has a more extended star formation history, with the lengthier timescale and higher proportion of young to old stars acting to mitigate the 'loss' of G-dwarfs from the present-day MDF.

In Figure 3, the star formation history of the Milky Way disk is compared with a more burst-dominated star formation history, which we here assume to be representative of ellipticals (or pure halo systems). The bulk of the stars in this burst model are born during the first $1.5 \mathrm{Gyr}$, after which the gas supply is depleted and the star formation rate declines rapidly. Conversely, it takes more than $5 \mathrm{Gyr}$ for the majority of stars to form in the disk model.

Figure 4 shows the predicted MDF of truly long-lived stars (black lines) and G-dwarfs (defined as having masses in the range $0.8<m / \mathrm{M}_{\odot}<1.4$ ) (grey lines) for the halo (dashed lines) and Milky Way (solid lines) models. The observed MDF of the NGC 5128 outer halo (Harris \& Harris 2002) is plotted as a histogram, where we have used the relation $[\mathrm{Fe} / \mathrm{H}]=[\mathrm{m} / \mathrm{H}]-0.25$ to transform the published values of $[\mathrm{m} / \mathrm{H}]$ into units of $[\mathrm{Fe} / \mathrm{H}]$. The black lines show the MDF that is expected if all G-dwarfs had lifetimes longer than the age of the galaxy (i.e., masses 


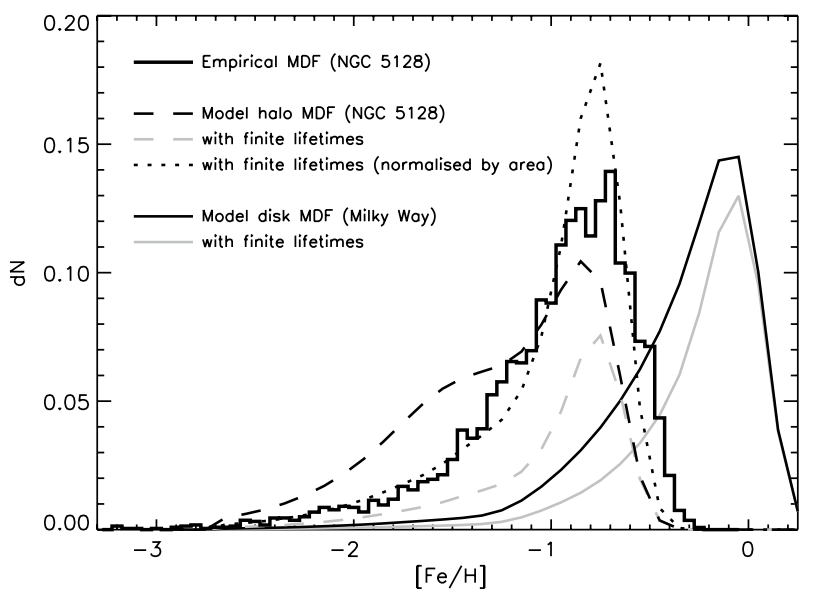

Figure 4 Predicted metallicity distributions compared with observations of the outer halo of NGC 5128 (Harris \& Harris 2000 histogram). We assume the relation $[\mathrm{Fe} / \mathrm{H}]=[\mathrm{m} / \mathrm{H}]-0.25$ when plotting the observed NGC 5128 MDF. Dashed curves correspond to a halo model, while solid curves represent the Milky Way model. The true MDF is indicated by black lines. Grey curves show the expected MDF of the G-dwarfs that remain in the sample at $t=15 \mathrm{Gyr}$ and thus demonstrate the loss of stars from the sample due to finite lifetimes. To illustrate the good fit, the dotted line presents the finite lifetime halo model results, normalized to the NGC $5128 \mathrm{MDF}$ sample size. Model predictions have been convolved with a Gaussian of $\sigma=0.1$ dex in metallicity.

$\left.\mathrm{m}<0.8 \mathrm{M}_{\odot}\right)$. These curves have been normalised to contain the same area as the histogram. The grey curves show the MDF that would be measured if the mass range of stars in the sample is $0.8<m / \mathrm{M}_{\odot}<1.4$. These have been normalised to reflect the loss of stars in each metallicity bin owing to evolution off the main sequence. The dotted line also shows the finite lifetime halo model MDF, scaled to illustrate the fit to the data. The loss of stars from a present-day sample of G-dwarfs can be seen to narrow the MDF and shift the distribution toward higher metallicities. While evident in both models, this behaviour is more pronounced in environments such as halos, where the bulk of the stars presumably formed more than $10 \mathrm{Gyr}$ ago.

By ignoring lifetime effects, one can overestimate the true mean metallicity of long-lived stars. To gauge the magnitude of this effect, we plot the cumulative metallicity distribution in Figure 5. For this particular halo model, mass-dependent stellar lifetimes serve to shift the median metallicity of the population by $\Delta[\mathrm{Fe} / \mathrm{H}]=+0.3$.

\section{Conclusions}

We have reproduced the metallicity distribution of nearby K-dwarfs using a model in which the Milky Way formed during two accretion episodes. Several key differences between this and other dual-phase infall models are (i) the assumption that the thin disk formed from metal-enriched $\alpha$-enhanced extragalactic material, (ii) the adoption of a new comprehensive set of stellar yields from Limongi et al. $(2000 ; 2002)$ for calculating the contribution to the enrichment of the ISM from massive stars, and (iii) a more gentle initial phase of (halo) star formation, resulting

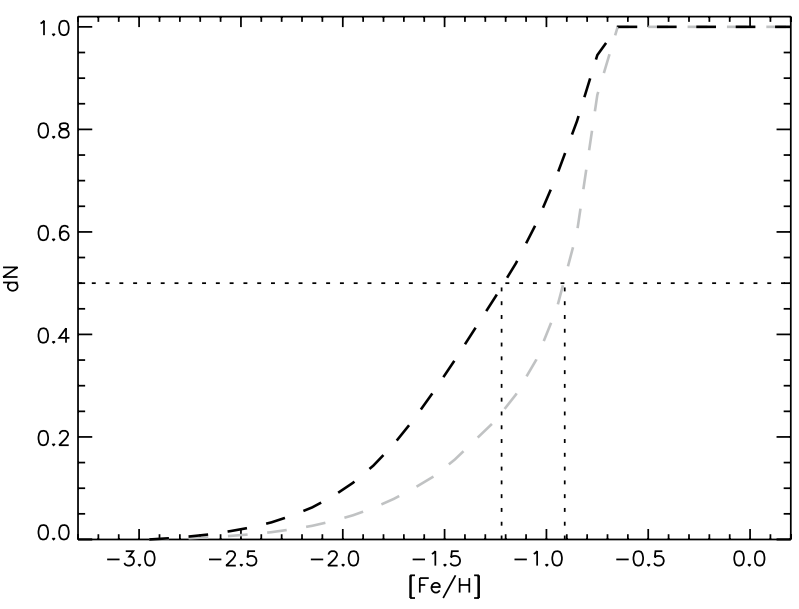

Figure 5 Cumulative metallicity distribution function for a model NGC 5128 halo. The black dashed line corresponds to the metallicity distribution of long-lived stars, while the grey dashed line shows the expected observed MDF for a sample of stars in the mass range $0.8<m / \mathrm{M}_{\odot}<1.4$, taking into account finite lifetimes. The median metallicity of a population corresponds to 0.5 on the vertical axis, as indicated by the horizontal dotted line. The evolution off the main sequence of older metal-poor G-dwarfs increases the median metallicity of the sample by $\sim 0.3$ dex.

in a shallower (but still observationally consistent) agemetallicity relation.

Taking the stellar halo as having $[\mathrm{Fe} / \mathrm{H}]<-1.2$ we predict that this component has an age of 12-13 Gyr and formed on a timescale of $\sim 1$ Gyr. If stars with metallicity $-1.0<[\mathrm{Fe} / \mathrm{H}]<-0.5$ are assigned to the thick disk, then the median age of this population is predicted to be $\sim 10$ Gyr with a greater scatter in ages $(\sim \pm 2$ Gyrs $)$ than for the halo. The age difference of $\sim 5 \mathrm{Gyr}$ between the halo and thin disk in our model is consistent with empirical estimates based upon white dwarf luminosity functions (Hansen et al. 2002).

The model presented in this paper was compared with a similar dual-phase infall model from Chiappini et al. (2001) that also reproduces the observed K-dwarf metallicity distribution. We show that even though differences in the rate of star formation in the early Galaxy might not be apparent in present-day observables such as the MDF, they do lead to different predictions for the ages and kinematics of the halo, thick, and thin disk components. This degeneracy is inherent in dual-infall models when applied to the modeling of three-component systems.

\section{Acknowledgments}

It is with pleasure that we thank the referees Sean Ryan and Eira Kotoneva for their very valuable comments on the manuscript. We also wish to thank Chris Flynn for many invaluable discussions. We are also grateful to Chris, Bill Harris and Gretchen Harris for providing their observational metallicity distribution functions prior to publication.

BKG acknowledges the financial support of the Australian Research Council through its Large Research Grant Program (Grant ID \#A00105171). 


\section{References}

Argast, D., Samland, M., Thielemann, F.-K. \& Gerhard, O. E. 2002, A\&A, 388, 842

Bazan, G. \& Mathews, G. J. 1990, ApJ, 354, 644

Carretta, E., Gratton, R. G. \& Sneden, C. 2000, A\&A, 356, 238

Chaboyer B., Sarajedini, A. \& Armandroff, T.E. 2000, AJ, 120, 3102

Chiappini, C., Matteucci, F. \& Gratton, R. 1997, ApJ, 477, 765

Chiappini, C., Matteucci, F. \& Romano, D. 2001, ApJ, 554, 1044

Durrell, P. R., Harris, W. E. \& Pritchet, C. J. 2001, AJ, 121, 2557

ESA, 1997, The Hipparcos and Tycho Catalogues, ESA, SP-1200

Fenner, Y. \& Gibson, B. K., in preparation

Fenner, Y., Gibson, B. K. \& Limongi, M. 2002, Ap\&SS, 281, 537

Gibson, B. K., Giroux, M. L., Penton, S. V., Stocke, J. T., Shull, J. M. \& Tumlinson, J. 2001, AJ, 547, 3280

Gilmore, G., Wyse, R. F. G. \& Jones, J. B. 1995, AJ, 109, 1095

Goswami, A. \& Prantzos, N. 2000, A\&A, 359, 191

Hansen, B. M. S., Brewer, J., Fahlman, G. G., Gibson, B. K., Ibata, R., Limongi, M., Rich, R. M., Richer, H. B., Shara, M. M. \& Stetson, P. B. 2002, ApJ, 574, L155

Ibukiyama, A. \& Arimoto, N. 2002, A\&A, 394, 927

Harris, G. L. H. \& Harris, W. E. 2000, AJ, 120, 2423

Harris, W. E. \& Harris, G. L. H. 2002, AJ, 123, 3108

Haywood, M. 2001, MNRAS, 325, 1365

Hou, J. L., Chang, R. \& Fu, C. 1998, in Pacific Rim Conference on Stellar Astrophysics, in K. L. Chan, K. L. Cheng, K. S. \& Singh, H. P., eds., (San Francisco: ASP), p. 143

Kotoneva, E., Flynn, C., Chiappini, C. \& Matteucci, F. 2002, MNRAS, 336, 879

Kroupa, P., Tout, C. A. \& Gilmore, G. 1993, MNRAS, 262, 545

Limongi, M., Straniero, O. \& Chieffi, A., 2000, ApJS, 129, 625
Limongi, M. \& Chieffi, A., 2002 PASA, 19, 246

Melendez, J., Barbuy, B. \& Spite, F. 2001, ApJ, 556, 858

McWilliam, A. 1997, ARAA, 35, 503

Norris, J. E. \& Ryan, S. G., 1991, ApJ, 380, 403

Prantzos, N. \& Silk, J. 1998, ApJ, 507, 229

Renzini, A. \& Voli, M. 1981, A\&A, 94, 175

Rocha-Pinto, H.-J. \& Maciel, W. J. 1996, MNRAS, 279, 447

Rocha-Pinto, H.-J. \& Maciel, W. J. 1997, A\&A, 325, 523

Romano, D., Matteucci, F., Salucci, P. \& Chiappini, C. 2000, ApJ, 539, 235

Ryan, S. G. Norris, J. E. \& Beers, T. C. 1996, ApJ, 471, 254

Salpeter, E. E. 1955, ApJ, 121, 161

Scalo, J. M. 1986, Fund. Cosm. Phys., 11, 1

Schaller, G., Schaerer, D., Meynet, G. \& Maeder, A. 1992, A\&AS, 96, 269

Sembach, K. R., Gibson, B. K., Fenner, Y. \& Putman, M. E. 2002, ApJ, 572, 178

Thielemann, F.-K., Nomoto, K., Hashimoto, M., 1993, in Prantzos, N., Vangiono-Flam, E. \& Casse, M., eds., Origin and Evolution of the Elements, Cambridge Univ. Press, Cambridge, p. 297

Tinsley, B. M. 1980, Fund. Cosm. Phys., 5, 287

Wakker, B. P., Howk, J. C., Savage, B. D., van Woerden, H., Tufte, S. L., Schwarz, U. J., Benjamin, R., Reynolds, R. J., Peletier, R. F. \& Kalberla, P. M. W. 1999, Nature, 402, 388

Woosley, S. E. \& Weaver, T. A. 1995, ApJS, 101, 181

Wyse, R. F. G. 2001, in J. G. Funes \& E. M. Corsini, eds., ASP Conf. Ser. 230, Galaxy Disks and Disk Galaxies, (San Francisco: ASP), 71

Wyse, R. F. G. \& Gilmore, G. 1995, AJ, 110, 2771 